\title{
Historia del trasplante de médula ósea en México.*
}

\author{
Historia de la Medicina
}

Guillermo J. Ruiz-Argüelles.

Director General, Centro de Hematología y Medicina Interna de Puebla.

Consultante en Hematología, Laboratorios Clínicos de Puebla, Puebla, Puebla, México.

\section{RESUMEN.}

El trasplante de células progenitoras hematopoyéticas (TCPH), se ha convertido en un recurso terapéutico imprescindible en la práctica moderna de la medicina. En México, la historia de los trasplantes de $\mathrm{CPH}$ puede dividirse en dos etapas. La primera etapa se inicia en el año de 1980 cuando se llevó a cabo el primer TCPH en México, que hicieron el Dr. Ricardo Sosa y sus colaboradores, en el Instituto Nacional de la Nutrición en la ciudad de México. Después de este trasplante, se hicieron algunos otros aislados en el Centro Médico Nacional, en el Hospital Universitario de Monterrey, en el propio Instituto Nacional de la Nutrición y en otros sitios, con resultados pobres. Esto dio como resultado que en varias instituciones médicas del país se suspendieran de manera transitoria los programas de TCPH. En México la práctica de los TCPH fue casi anecdótica hasta antes de 1995.

La segunda etapa se inició a partir de 1995, con la llegada de algunos médicos entrenados en la práctica de los TCPH. Otra causa por la que se reactivaron los programas de TCPH, fue la evolución de los

*Artículo publicado en Rev Hematol 2004; 5:80-85. Reproducido con autorización. conocimientos en esta área: a) se comenzaron a usar CPH de sangre periférica en vez de médula ósea; $b$ ) se hicieron simplificaciones de los métodos para llevar a cabo los trasplantes, y c) se inició la práctica de los alotrasplantes con esquemas de acondicionamiento no mieloablativo.

Los trasplantes alogénicos no mieloablativos (TANM) han encontrado un terreno muy fértil para realizarse en países en desarrollo, entre ellos en México, ya que son considerablemente más baratos, más simples e igualmente eficientes. En el año de 1999 se inició un programa de TANM en Monterrey y Puebla usando un esquema novedoso, accesible y barato, que emplea fludarabina, ciclofosfamida y busulfán. Este esquema "mexicano" ha mostrado ser útil para trasplantar a niños, adultos y sujetos añosos y ha producido resultados similares a los de los esquemas "tradicionales" de acondicionamiento pretrasplante. La accesibilidad y eficiencia de este método ha dado como resultado que varias instituciones del país, entre ellas el Centro Médico la Raza del IMSS y el Instituto Nacional de Cancerología, lo usen y que además, se haya adoptado como el método de referencia por el grupo LACOHG (Latin-American Cooperative Onco-Hematology Group).

Solicitud de sobretiros: Dr. Guillermo J. Ruiz-Argüelles, Laboratorios Clínicos de Puebla, Blvd. Díaz Ordaz No. 808, Col. Anzures, C.P. 72530, Puebla, Puebla, México. Tel. (222) 243-81-00 Aceptado para publicación el 9/Septiembre/2005. 


\section{GJ Ruiz-Argüelles.}

Los trasplantes de células de cordón umbilical se han llevado a cabo en el Hospital Universitario de Monterrey, en el Centro de Hematología y Medicina Interna de Puebla, en el Hospital Ángeles de la ciudad de México y probablemente en otras instituciones. Se han organizado bancos de células placentarias en el Centro Nacional de la Transfusión Sanguínea, el Hospital Universitario de Monterrey, la Unidad de Criopreservación de Puebla y otras instituciones privadas.

La práctica de los TCPH en México, iniciada en 1980, estuvo estancada durante un tiempo pero, para fortuna de los pacientes quienes requieren de estos tratamientos, ha tenido un crecimiento más rápido en los últimos años. En 2003, se informaron resultados de 1337 pacientes trasplantados en todo el país.

(Rev Biomed 2005; 16:207-213)

Palabras clave: Historia de la Medicina, trasplante de células hematopoyéticas, México.

\section{SUMMARY.}

History of Bone Marrow Transplant in Mexico.

The transplant of parent haematopoyetic cells (TPHC) has become an indispensable therapeutic resource in modern medicine. In Mexico, TPHC transplants can be divided into two stages. The first stage began in 1980 when Dr. Ricardo Sosa and his team performed the first TPHC transplant at the Instituto Nacional de la Nutrición in Mexico City. After this transplant, some others were performed at the Centro Medico Nacional, the Hospital Universitario de Monterrey, México, and at the Instituto Nacional de la Nutrición itself, among other institutions, all with poor results. This resulted in the temporary suspension of TPHC programs in several hospitals throughout the country. The practice of TPHC became almost anecdotal until 1995. In that year the second stage began with the arrival of some doctors already trained in TPHC. Another factor causing the reactivation of TPHC programs was the development of knowledge in this area: a) PHC from peripheral blood was used instead of bone marrow; b) methods to carry out transplants were simplified, and; c) allotransplant with non-mieloblative conditioning schemes were introduced.

Non-mieloblative allogeneic transplants (NMAT) have found fertile ground in developing countries, Mexico among them, since they are much cheaper, simpler, and just as efficient. In 1999, a NMAT program began in Monterrey and Puebla using a new, cheap, and accessible scheme employing fludarabine, ciclofosfamide, and busulfan. This "Mexican" scheme has proven useful in transplants for children, adults, and elderly patients, and it has produced results similar to the "traditional" pre-transplant conditioning schemes. The accessibility and efficiency of this method have resulted in its adoption by several of the national institutions, including the Centro Médico de la Raza of the Mexican Institute of Social Security and the Instituto Nacional de Cancerología, and it has even been adopted as a reference method by the LACOHG (Latin American Cooperative OncoHaemotology Group).

Umbilical cord cell transplants have taken place at the University Hospital of Monterrey, Mexico; the Haematology and Internal Medicine Center of Puebla; the Angeles Hospital of Mexico City and probably in other institutions. Placenta cell banks have been organized at the National Center of Blood Transfusion, the University Hospital of Monterrey, and the Cryopreservation Unit of Puebla, and in other private institutions.

The practice of TPHC in Mexico began in 1980, was stalled for some time, but luckily for the patients who require this treatment, it has recently restarted. In 2003, results of 1337 transplanted patients were reported nationwide.

Key words: History of Medicine, haematopoyetic cell transplants, Mexico.

\section{INTRODUCCIÓN.}

El trasplante de células progenitoras hematopoyéticas (TCPH), también llamado trasplante de células hematopoyéticas totipotenciales, en alguna

\section{Revista Biomédica}




\section{Historia del trasplante de médula ósea en México.}

época se conoció como trasplante de médula ósea, ya que de este sitio era de donde se obtenían preferentemente las $\mathrm{CPH}$. Este procedimiento se ha convertido en un recurso terapéutico imprescindible en la práctica moderna de la medicina.

Como resultado de la evolución de los conocimientos sobre el TCPH, se han roto varios dogmas que por mucho tiempo obstaculizaron el desarrollo de esta área de la medicina. Ahora se sabe que: a) para la toma de injerto exitosa de las $\mathrm{CPH}$ no se requiere destrucción de la médula ósea del receptor; b) las CPH crean su propio espacio en la médula del receptor por medio de efectos de injerto contra huésped; c) diversos tumores se pueden erradicar merced al efecto de injerto contra tumor; d) es posible llevar a cabo trasplantes de $\mathrm{CPH}$ alogénicas de manera extrahospitalaria; e) se pueden hacer alotrasplantes en sujetos añosos o debilitados; f) es posible hacer trasplantes alogénicos sin transfusiones de eritrocitos ni de plaquetas y g) es posible disminuir los costos de los alotrasplantes de manera significativa.

Todos estos cambios han dado como resultado la disponibilidad de los TCPH a un mayor número de pacientes, tanto en México como en otros países en desarrollo, ofreciendo así, en algunos casos, una opción verdaderamente curativa para pacientes quienes hasta hace poco no podían tener acceso a estos tratamientos modernos. En nuestro país, la historia de los trasplantes de $\mathrm{CPH}$ puede dividirse en dos etapas, que se analizarán por separado.

\section{LA PRIMERA ETAPA.}

En el año de 1980 se llevó a cabo el primer TCPH en México, un verdadero trasplante de médula ósea, que hicieron el Dr. Ricardo Sosa y sus colaboradores, en el Instituto Nacional de la Nutrición en la ciudad de México (1). El Dr. Sosa acababa de llegar de su entrenamiento en TCPH en Seattle, con el Dr. E. Donnall Thomas, quien en 1990 fue merecedor del Premio Nobel de Medicina por sus contribuciones en esta área de la medicina. Después de este trasplante, se hicieron algunos otros aislados en el Centro Médico Nacional, en el Hospital Universitario de Monterrey, en el propio Instituto
Nacional de la Nutrición y en otros sitios, con resultados pobres. Esto dio como resultado que en varias instituciones médicas del país se suspendieran de manera transitoria los programas de TCPH, mientras que en los Estados Unidos de Norteamérica y en otros países desarrollados, la actividad de los programas crecía de manera exponencial. En México la práctica de los TCPH fue casi anecdótica hasta antes de 1995 (1-4).

\section{LA SEGUNDA ETAPA.}

Se inició a partir de 1995, con la llegada de algunos médicos entrenados en la práctica de los TCPH, lo que reactivó algunos de los programas de TCPH en el país e inició otros (5-24). Otra causa por la que se reactivaron en algunas instituciones y se iniciaron en otras la actividad más intensa de los programas de TCPH, fue la evolución de los conocimientos en esta área: a) se comenzaron a usar $\mathrm{CPH}$ de sangre periférica en vez de médula ósea; b) se hicieron simplificaciones de los métodos para llevar a cabo los trasplantes, y c) se inició la práctica de los alotrasplantes con esquemas de acondicionamiento no mieloablativo.

Las CPH de sangre periférica son más fáciles y baratas de obtener y permiten una recuperación más rápida de la hematopoyesis del receptor, lo que se traduce en disminución de costos de hospitalización, medicamentos, transfusiones, etc. En el caso de los trasplantes autólogos, no hay duda de que las $\mathrm{CPH}$ periféricas son mucho mejores que las $\mathrm{CPH}$ de médula ósea (8-10), lo que aún no es totalmente claro para los alotrasplantes.

Por otro lado, el crecimiento exponencial en todo el mundo de los TCPH alogénicos usando esquemas de acondicionamiento no mieloablativo, ha incidido en el crecimiento de la actividad de los trasplantes en México y en otros países en desarrollo. Los trasplantes alogénicos no mieloablativos (TANM) han encontrado un terreno muy fértil para desarrollarse en países en desarrollo, entre ellos en México, ya que son considerablemente más baratos, más simples e igualmente eficientes. Adicionalmente, los TANM no precisan de instalaciones médicas 


\section{GJ Ruiz-Argüelles.}

sofisticadas, como cámaras de flujo laminar o de presión positiva, de las que en el país no existen más de 3 ó 4, aunque sí de una preparación médica adecuada e infraestructura de laboratorio y banco de sangre suficientes (11-29).

En el año de 1999 se inició un programa de TANM en Monterrey y Puebla usando un esquema novedoso, accesible y barato, que emplea fludarabina, ciclofosfamida y busulfán (20). Este esquema "mexicano" (23) ha mostrado ser útil para trasplantar a niños, adultos y sujetos añosos y ha producido resultados similares a los de los esquemas "tradicionales" de acondicionamiento pre-trasplante en varios padecimientos como leucemia granulocítica crónica, leucemia aguda mieloblástica, hipoplasia medular, leucemia linfoblástica, enfermedad de Hodgkin, etc. El esquema se puede emplear también para hacer trasplantes de células placentarias. La accesibilidad y eficiencia de este método ha dado como resultado que varias instituciones del país, entre ellas el Centro Médico la Raza del IMSS y el Instituto Nacional de Cancerología, lo usen y que además, se haya adoptado como el método de referencia por el grupo LACOHG (Latin-American Cooperative Onco-Hematology Group) para trasplantar sujetos con leucemias mieloides crónicas y agudas mieloblásticas. Se ha empleado ya en Colombia, Venezuela, Brasil y en otros sitios del país, con resultados similares a los descritos inicialmente en Monterrey y en Puebla (29). El hecho de que 3 trabajos publicados sobre la simplificación de los métodos para llevar a cabo los TCPH en el país hayan sido acreedores al Premio CARSO-FUNSALUD que cada dos años se concede a trabajos hechos en el país relacionados con trasplantes de cualquier órgano, indica, entre otras cosas, el interés que ha habido en los últimos años en México por el trasplante de células hematopoyéticas.

Los resultados de los TANM hechos en todo el mundo en comparación a los trasplantes convencionales han mostrado que tienen menor morbimortalidad, eficacia anti-tumoral similar, incidencia similar de enfermedad de injerto contra huésped y, en algunos países, sobre todo en aquellos en vías de desarrollo, costos menores, lo que los hace más accesibles y en algunos casos, los convierte en la única opción terapéutica verdaderamente eficaz.

\section{EL INFORME DE LOS TRASPLANTES HECHOS EN MÉXICO EN REGISTROS INTERNACIONALES.}

Como resultado del crecimiento de la actividad de los TCPH en el país, se han comenzado a registrar algunos de los trasplantes hechos en México en registros internacionales como el IBMTR / ABMTR (International Bone Marrow Transplantation Registry / Autologous Bone Marrow Transplantation Registry). Para enero de 2004, había siete instituciones mexicanas registradas en el IBMTR/ABMTR (cuadro 1). Es deseable que todas las instituciones del país involucradas en estos tratamientos inscriban sus datos en los registros internacionales. Otras instituciones que también llevan a cabo TCPH pero que no envían sus datos a este registro aparecen en la cuadro 2. Es muy posible que en este cuadro se omitan, no deliberadamente, otras instituciones mexicanas que llevan a cabo estos procedimientos terapéuticos.

\section{Cuadro 1}

Instituciones mexicanas que registran los datos de sus trasplantes en el IBMTR / ABMTR

\begin{tabular}{|c|c|}
\hline Institución & Ubicación \\
\hline Centro Médico del Norte & Monterrey \\
\hline Centro Médico La Raza & México DF \\
\hline Hospital Angeles-Lomas & México DF \\
\hline Hospital San José & Monterrey \\
\hline Instituto Nacional de Cancerología & México DF \\
\hline Hospital Universitario de Monterrey & Monterrey \\
\hline \multicolumn{2}{|l|}{ Centro de Hematología y Medicina } \\
\hline Interna & Puebla \\
\hline
\end{tabular}

\section{EL NÚMERO DE TCPH HECHOS EN MÉXICO.}

No existe información precisa sobre cuántos TCPH se han hecho en México. En 2003, el Dr. Alejandro Limón organizó en la ciudad Puebla un encuentro de directores de programa de TCPH en

\section{Revista Biomédica}


Historia del trasplante de médula ósea en México.

México en el que se informaron resultados de 1337 pacientes trasplantados en todo el país (30-31). En el programa conjunto de TCPH Monterrey / Puebla, para agosto de 2004 se habían llevado a cabo más de 250 trasplantes de células hematopoyéticas (8-29).

\section{Cuadro 2}

Otras instituciones mexicanas que llevan a cabo ТСРН.

\begin{tabular}{ll}
\hline \multicolumn{1}{c}{ Institución } & Ubicación \\
\hline Centro Médico Nacional & México DF \\
Centro Médico M. Avila Camacho & Puebla \\
Centro Médico de Occidente & Guadalajara \\
Instituto Nacional de Ciencias Médicas & \\
yde la Nutrición “Dr. Salvador Zubirán” & México DF \\
Hospital 20 de Noviembre & México DF \\
Hospital Infantil de México & México DF \\
\hline
\end{tabular}

\section{LOS TRASPLANTES DE CÉLULAS DE CORDÓN UMBILICAL.}

Se han llevado a cabo en el Hospital Universitario de Monterrey, en el Centro de Hematología y Medicina Interna de Puebla, en el Hospital Angeles de la ciudad de México y probablemente en otras instituciones. Sólo las dos primeras han hecho publicaciones formales de trasplantes de células placentarias. Se han organizado bancos de células placentarias en el Centro Nacional de la Transfusión Sanguínea, el Hospital Universitario de Monterrey, la Unidad de Criopreservación de Puebla y otras instituciones privadas. Las dos primeras instituciones tienen bancos "públicos" de células placentarias, que es la conducta ideal y el futuro de estas organizaciones. Se han hecho también algunos trasplantes hematopoyéticos con células placentarias obtenidas de bancos internacionales (28).

\section{LAS PUBLICACIONES MEXICANAS SOBRE TCPH.}

De manera similar a lo que ocurre en otras áreas de la medicina, las publicaciones generadas en el país derivadas de la práctica de los TCPH son pocas. Sólo en algunos centros de TCPH se han generado publicaciones. Al buscar trabajos en la base de datos
PubMed con las palabras: México, marrow, transplantation, se encontraron trabajos publicados en revistas con revisión por pares de las siguientes instituciones: Centro Médico La Raza, Centro Médico Nacional, Centro Médico de Occidente, Instituto Nacional de Cancerología, Instituto Nacional de Nutrición, Hospital Universitario de Monterrey y Centro de Hematología y Medicina Interna de Puebla. Es interesante que el mayor número de estas publicaciones en la segunda etapa de la práctica de los TCPH ha sido generado en las instituciones localizadas fuera de la ciudad de México y que las publicaciones nacionales sobre el tema han sufrido un incremento casi exponencial a partir de la introducción de las simplificaciones a los métodos para llevar a cabo los TCPH en el país. La figura 1 resume estos datos (30).

\section{CONCLUSIONES.}

La práctica de los TCPH en México, iniciada en 1980, estuvo estancada durante un tiempo pero, para fortuna de los pacientes quienes requieren de estos tratamientos, ha tenido un crecimiento más rápido en los últimos años; sin embargo, sigue siendo limitada. El uso de esquemas de preparación simplificados para los trasplantes autólogos, de esquemas de

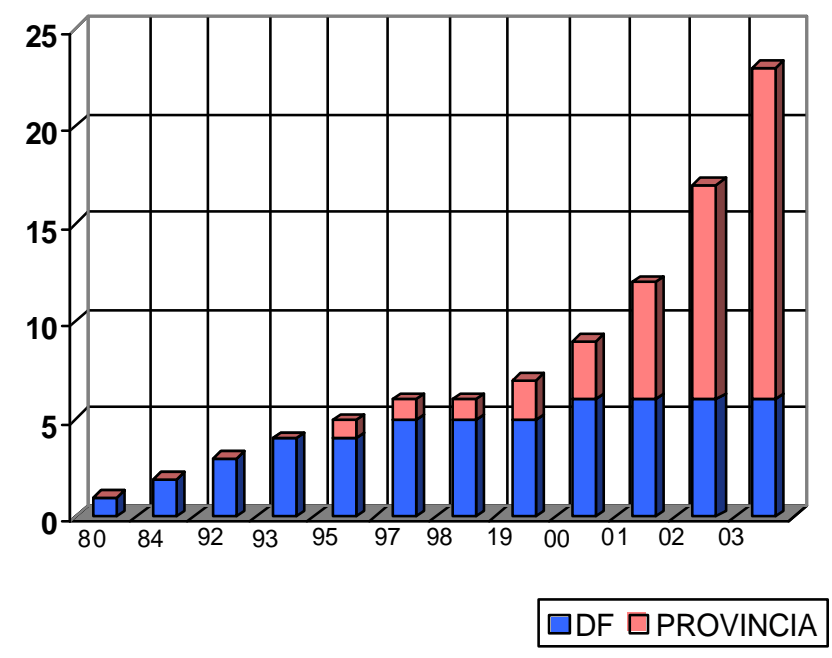

Figura 1.- Curva de frecuencias acumuladas de las publicaciones mexicanas sobre trasplante de células hematopoyéticas en revistas con revisión por pares, entre 1980 y 2003 (30).

Vol. 16/No. 3/Julio-Septiembre, 2005 


\section{GJ Ruiz-Argüelles.}

acondicionamiento no mieloablativo para los trasplantes alogénicos y de CPH de sangre periférica para ambos, han permitido ofrecer este recurso terapéutico a un mayor número de pacientes. Es deseable que todos los médicos quienes hacen TCPH informen sus resultados a los registros internacionales y que participen más activamente en labores de investigación y de difusión de conocimientos sobre el tema.

\section{REFERENCIAS.}

1.- Sosa-Sánchez R, Córdova MS, Labardini JR, Chávez-Peón F. Trasplante de médula ósea en anemia aplástica. Reporte del primer caso en México. Rev Invest Clin Mex 1980; 32:4955.

2.- León-Rodríguez E, Sosa-Sánchez R, Gómez E, OchoaSosa C.: Bone marrow transplantation in aplastic anemia: Experience at a Mexican institution. Rev Invest Clin Mex 1993;45:559-64.

3.- León-Rodríguez E, Sosa-Sánchez R. Bone marrow transplantation in México. Report of the first succesful graft in acute myeloblastic leukemia. Grupo de trasplante medular óseo del INNSZ. Rev Invest Clin Mex 1992; 44:383-6.

4.- Morales-Polanco MR, Pizzuto-Chávez J. Bone marrow transplant in aplastic anemia. Current status and review of the first allogeneic transplants in México. Gac Med Mex 1984; 120:49-57.

5.- Delgado-Lamas JL, Garcés-Ruiz OM, Rubio-Jurado B. Hematopoietic cell transplantation: Experience at the Western National Medical Center. Rev Invest Clin 2000; 52:234-40.

6.- Vela-Ojeda J, Tripp-Villanueva F, Sánchez-Cortés E, AyalaSánchez M, Rosas-Cabral A, Esparza MG, et al. Allogeneic bone marrow transplantation for chronic myeloid leukemia: a single center experience. Arch Med Res 2000; 31:206-09.

7.- Reynoso-Gómez EE, Navarrete-Herrera Jr, Salinas-Rojas V, Sobrevilla-Calvo P, Miranda-López E. Experience with 22 bone marrow donors. Rev Invest Clin Mex 1997; 49:465-68.

8.- Ruiz-Argüelles GJ, Gómez-Rangel D, Ruiz-Delgado GJ, Ruiz-Argüelles A, Pérez-Romano B, Rivadeneyra L. Results of an autologous non-cryopreserved, unmanipulated peripheral blood hematopoietic stem cell transplant program: A single institution, 10-year experience. Acta Haematologica
2003; 110: 179-83.

9.- Ruiz-Argüelles GJ, Lobato-Mendizábal E, Ruiz-Argüelles A, Pérez-Romano B, Arizpe-Bravo D, Marín-López A. Noncryopreserved unmanipulated hematopoietic peripheral blood stem cell autotrasplant program: Long term results. Arch Med Res 1999; 30:380-4.

10.- Ruiz-Argüelles GJ, Ruiz-Argüelles A, Pérez-Romano B, Marín-López A, Larregina-Díez A, Apreza-Molina MG. Filgrastim-mobilized peripheral-blood stem cells can be stored at 4 degrees and used in autografts to rescue highdose chemotherapy. Am J Hematol 1995; 48:100-3.

11.- Ruiz-Argüelles GJ. Allogeneic stem cell transplantation using non-myeloablative conditioning regimens: Results of the Mexican approach. Int J Hematol 2002; 76 (Suppl 1): 3769.

12.- Ruiz-Argüelles GJ, Ruiz-Argüelles A, Gómez-Almaguer D, López-Martínez B, Abreu-Díaz G, Bravo G, Jaime-Pérez JC. Features of the engraftment of allogeneic hematopoietic stem cells using reduced-intesity conditioning regimens. Leukemia Lymph 2001;42:145-50.

13.- Ruiz-Argüelles GJ, Gómez-Almaguer D, López-Martínez B, Ponce-de-León S, Cantú-Rodríguez OG, Jaime-Pérez JC. Non cytomegalovirus-related deaths after non-ablative stem cell allografts. Hematology 2002; 7:95-9.

14.- Ruiz-Argüelles GJ, Gómez-Rangel JD, Ponce-de-León S, González-Déctor L, Reyes-Núñez V, Garcés-Eisele J. The Mexican schedule to conduct allogeneic stem cell transplantation is related to a low risk of cytomegalovirus reactivation and disease. Am J Hematol 2004; 75;200-4.

15.- Ruiz-Argüelles GJ, López-Martínez B, Santellán-Olea MR, Abreu-Díaz G, Reyes-Núñez V, Ruiz-Argüelles A, GarcésEisele J. Follow up of hemopoietic chimerism in individuals given allogeneic hemopoietic stem cell allografts using an immunosuppressive, non-myeloablative conditioning regimen: A prospective study in a single institution. Leukemia Lymph 2002; 43:1509-11.

16.- Ruiz-Argüelles GJ, Gómez-Almaguer D, López-Martínez B, Cantú-Rodríguez OG, Jaime-Pérez JC, González-Llano O. Results of an allogeneic non-myeloablative stem cell transplantation program in patients with chronic myelogenous leukemia. Haematologica 2002; 87: 894-6.

17.- Ruiz-Argüelles GJ, Gómez-Almaguer D, Gómez Rancel JD, Vela-Ojeda J, Cantú-Rodríguez OG, Jaime-Pérez JC, et al. Allogeneic hematopoietic stem cell transplantation with non-

\section{Revista Biomédica}




\section{Historia del trasplante de médula ósea en México.}

myeloablative conditioning in patients with acute leukemia eligible for conventional allografting: A prospective study. Leukemia Lymphoma 2004; 45:1191-5.

18.- Gómez-Almaguer D, Ruiz-Argüelles GJ, Tarín-Arzaga LC, González-Llano O, Jaime-Pérez JC, López-Martínez B, etal. Reduced-intensity stem cell transplantation in children and adolescents: The Mexican experience. Biol Blood Marrow Transpl 2003; 9:157-61.

19.- Ruiz-Argüelles GJ, López-Martínez B, Gómez-Rangel D, Estrada E, Marín-López A, Bravo-Hernández G, et al. Decreased transfusion requirements in patients given stem cell allografts using a non-myeloablative conditioning regimen: A single institution experience. Hematology 2003; 8: 151-4

20.- Gómez-Almaguer D, Ruiz-Argüelles GJ, Ruiz-Argüelles A, González-Llano O, Cantú OE, Hernández NE. Hematopoietic stem cell allografts using a non-myeloablative conditioning regimen can be safely performed on an outpatient basis. Bone Marrow Transpl 2000; 25:131-3.

21.- Ruiz-Argüelles GJ, Gómez-Almaguer D, Ruiz-Argüelles A, González-Llano O, Cantú OG, Jaime-Pérez JC. Results of an outpatient-based stem cell allotransplant program using non-myeloablative conditioning regimens. Am J Hematol 2001;66:241-4.

22.- Ruiz-Argüelles GJ, López-Martínez B, López-Ariza B. Succesful allogeneic stem cell transplantation with nonmyeloablative conditioning in patients with relapsed Hodgkin's disease following autologous stem cell transplantation. Arch Med Res 2003; 34:242-5.

23.- Ruiz-Argüelles GJ. "The Mexican approach" to conduct non-myeloablative stem cell transplantation. Eur J Haematol 2001;67:5-6.

24.- Gómez-Almaguer D. The simplification of the stem cell transplantation procedures in developing countries has resulted in cost-lowering and availability to more patients. Int J Hematol 2002; 76 (Suppl 1): 380-2.

25.- Ruiz-Argüelles GJ, Gómez-Rangel D, Sánchez-Anzaldo J, Ruiz-Argüelles A, Porras-Ramírez G, Luis-López A. Trasplante de células de cordón umbilical: Informe de dos casos. Medicina Univ 2002; 4:233-5.

26.- Ruiz-Argüelles GJ. Non-myeloablative bone marrow transplantation. Arch Med Res 2003; 34:554-7.

27.- Ruiz-Argüelles GJ, Gómez-Almaguer D. Breaking dogmata to help patients: Non-myeloablative hematopoietic stem cell transplantation. Expert Opin Biol Ther 2004; 4:16939.

28.- Ruiz-Argüelles GJ, Reyes-Núñez V, Garcés-Eisele J, Warwick RM, McKenna L, Ruiz-Reyes G, et al. Acquired hemoglobin $\mathrm{S}$ trait in an adult patient with secondary acute myelogenous leukemia allografted with matched unrelated umbilical cord blood cells. Enviado a publicación.

29.- Ruiz-Argüelles GJ, Gómez-Almaguer D, Gómez-Rangel JD, Vela-Ojeda J, Karduss A, Cantú-Rodríguez OG, et al. Allogeneic hematopoietic stem cell transplantation with nonmyeloablative conditioning in patients with acute myelogenous leukemia elegible for conventional allografting: a prospective study. Leuk Lymphoma 2004; 45: 1191-5.

30.- Ruiz-Argüelles GJ, Gómez-Almaguer D, Gómez-Morales E. Trasplante de células progenitoras hematopoyéticas. En Góngora-Biachi R. (editor) Hematología: Actualización 2004. Mérida: Ediciones de la Agrupación Mexicana para el Estudio de la Hematología A.C. 2004. pp. 139-48.

31.- Limón-Flores JA, González-Pérez ME, Lobato-Tolama RD, Valderrama-Bazán LG. Jornadas de trasplante hematopoyético en el IMSS de Puebla. Resultados. Rev Hematol 2004; 5 (Suppl 1): S11. 